\title{
Doação de sangue: uma visão de marketing
}

\author{
Blood donation: a marketing perspective
}

\section{Pontifícia Universidade Católica do Rio Grande do Sul, Porto Alegre, Brasil. \\ Correspondência \\ S. T. Ludwig \\ Pontifícia Universidade \\ Católica do Rio Grande \\ do Sul. Av. Iguaçu 206, \\ apto. 401, Porto Alegre, RS \\ 90470-430, Brasil. \\ silvia_ludwig@terra.com.br}

\section{Abstract}

This paper emphasizes how marketing can make a difference in repeat donations by volunteer blood donors, since the greatest challenge for health institutions is to maintain and increase blood donation. In this context, understanding volunteer donors' motivations is highly important, and the studies reported here demonstrate that several variables are relevant to blood donation. The huge number of patients in need of blood transfusions and the lack of sufficient blood and blood products justify the interest in this study, considering both donors' and blood banks' perspectives. Moreover, recognition of the importance of actions and orientation for donors is fundamental for developing a marketing strategy. It is thus relevant for health institutions to identify donors' actual needs and wishes.

Blood Donors; Social Marketing; Strategies
Silvia Terra Ludwig 1

Alziro César de Morais Rodrigues 1

\section{Introdução}

Considerando o crescente aumento da população e os importantes avanços tecnológicos ocorridos no campo da Medicina, o setor de assistência hemoterápica apresenta-se como área fértil para o desenvolvimento de estudos mercadológicos. Nesse cenário, um estudo desta natureza é relevante por tratar-se de um mercado carente e com múltiplas necessidades.

Observa-se a importância da narrativa econômica nesta área, pois para a prestação de serviços hemoterápicos é necessário empregar pessoas e aplicar recursos. Desta forma, devese evidenciar que o setor, composto pela hemorrede pública estadual e municipal (hemocentros e suas unidades), complementada pela iniciativa privada (serviços de hemoterapia e bancos de sangue) é formado por instituições orientadas para objetivos e dedicadas a produzir produtos e serviços para satisfazer as necessidades dos clientes, que podem ser consumidores, usuários, associados, contribuintes.

Assim, evidencia-se que a partir do conhecimento das necessidades dos doadores os hemocentros, serviços de hemoterapia e bancos de sangue podem aprimorar suas relações com estes. Pois, à medida que se tornam mais dinâmicas as necessidades dos doadores, aumentam as exigências da capacidade de resposta das instituições, com vistas a disponibilização de recursos humanos e materiais. Nesse senti- 
do, vai-se ao encontro do que preconiza a Política Nacional de Sangue e Hemoderivados do Ministério da Saúde, que visa aumentar o número de doadores. A meta é elevar o percentual de doadores para $2,2 \%$ da população em 2005 e atingir 3,0\% até 2007. "Esse é o nível recomendado pela Organização Mundial da Saúde (OMS) e o limite que nos dá mais segurança e que nos permitirá contar com estoques reguladores" 1.

Nesse contexto, destaca-se que o propósito do marketing é atender e satisfazer necessidades e desejos. Entretanto, a estratégia de marketing de serviços exige não apenas marketing externo, mas também marketing interno para motivar os colaboradores e marketing interativo visando à qualidade da interação doadorinstituição. Esta estratégia permite que a instituição aprimore seus serviços e crie valor agregado para o doador. Pois estes avaliam a qualidade dos serviços de acordo com alguns critérios: aspectos tangíveis, competência, velocidade de atendimento, flexibilidade, credibilidade, segurança, acesso e custo, entre outros 2.

Em uma unidade hemoterápica ocorre que o doador e os colaboradores interagem para criar o serviço. Os prestadores de serviços necessitam trabalhar eficazmente para criar valor superior durante esses contatos. A intenção deste artigo, portanto, é contribuir com o Programa Nacional de Doação Voluntária de Sangue (PNDVS) e sensibilizar as instituições para aprimorar a captação de doadores.

\section{Marketing na doação de sangue}

Recentes mudanças na percepção do público sobre os riscos e benefícios da transfusão de sangue têm afetado a prática da doação de sangue. Estudos sobre a escassez de sangue motivaram as instituições a implementar estratégias de marketing 3,4 . A capacidade das instituições para o atendimento à população depende da sua habilidade em compreender e dominar esse ambiente.

Destaca-se que a doação de sangue no Brasil é um ato voluntário, conforme disposto na Constituição da República e na Portaria $n .343$ (Diário Oficial da União 2002; 19 fev) que estabelecem que não é admitido qualquer tipo de remuneração para a doação. A doação altruísta é, assim, a fonte de matéria-prima das unidades hemoterápicas.

Segundo o Ministério da Saúde, em torno de 3,5 milhões de bolsas de sangue são coletadas anualmente no Brasil 5. O maior desafio enfrentado pelas instituições de saúde, portanto, é manter e incrementar a doação de sangue, pois os doadores representam apenas $1,7 \%$ da população brasileira 1 .

Com o intuito de elevar estes números, o PNDVS foi instituído pelo Governo Federal, Ministério da Saúde, em novembro de 1998, tendo por objetivo envolver a sociedade brasileira, levando-a a participar ativamente do processo de doação de sangue de forma consciente e responsável, através de ações educativas e de mobilização social, visando a garantia da quantidade adequada à demanda do país e a melhoria da qualidade do sangue, componentes e derivados 1 .

No caso do doador voluntário, que vai doar levado por vários motivos, desde a dádiva, o altruísmo e até agradecimento por já ter sido ajudado dessa mesma forma, mais importante se torna este estudo. O doador voluntário, em um primeiro momento, vai doar guiado ou por um pedido, que pode ser de um amigo ou parente que esteja necessitando de sangue, ou por uma fonte, que pode ser a indicação de alguém que já doou nesse lugar. A percepção dessa fonte, um indivíduo fora de seu ambiente familiar, geralmente sob pressão de tempo, provavelmente cansado e talvez fragilizado por doar para um familiar que está necessitado, demonstra a importância de converter essa experiência em algo que acrescente valor para o doador a ponto de que este se torne uma boa fonte de referências 6 .

As percepções do usuário sobre o atendimento podem servir para diferenciar a oferta em serviços semelhantes. Seres humanos provavelmente são guiados por motivos racionais que influenciam suas decisões de doar sangue. Para Solomon et al. 7, confiança é um fator importante, porque envolve escolher tendo a convicção de que o resultado depende das ações da instituição, que o beneficiarão ou pelo menos não serão nocivas. Se os colaboradores da instituição falharem em realizar os procedimentos, levando em consideração que a implementação desse serviço exige um contato pessoal entre o prestador do serviço e o doador, isso resultará em danos para os doadores.

Na doação de sangue, onde o cliente não vai comprar um serviço, mas, sim, doar e possibilitar uma prestação de serviço que vai, ao final, beneficiar outra pessoa, a observação detalhada dos itens acima e o conseqüente aproveitamento dessas informações pode influenciar a 
prestação de serviços particularmente nas tarefas de contato com o doador. Conforme Andaleeb \& Basu 8, a verdadeira decisão para doar sangue envolve a escolha racional de confiar nas instituições e nos métodos que utilizam.

\section{Hemocentros, serviços de hemoterapia} e bancos de sangue

Estas instituições têm uma importância social muito grande. Primeiro, por atender pacientes, que, sem reposição sangüínea, não sobreviveriam. Segundo, devido a determinações legais, um hospital não pode funcionar sem uma unidade hemoterápica. Para a indústria, que recebe o excedente, isto é, o que não foi utilizado na transfusão, a falta de doadores se transforma em falta de matéria-prima, gerando produção menor, que não atende a demanda.

Porém, verifica-se que a preocupação maior de cada unidade hemoterápica é com o atendimento das necessidades dos pacientes. Nos casos de urgências (acidentes graves) é de suma importância o pronto atendimento. Existem, também, pacientes com doenças crônicas, graves, que necessitam fazer transfusões regularmente, casos das hemodiálises, onde a busca por doadores é enorme.

Assim, a busca do doador voluntário e habitual se deve principalmente à segurança, mas também à economia, pois doadores testados e retestados significam bolsas de sangue com margem maior de segurança para o receptor e menos exames sorológicos desprezados. Se toda a população realizasse esses exames com freqüência, estes teriam um caráter preventivo e muitas doenças poderiam ser evitadas ou acompanhadas 6 . Nesse contexto, torna-se relevante destacar as variáveis consideradas importantes pelo doador.

\section{Variáveis importantes na doação de sangue}

Estudos demonstram que diversas variáveis são importantes na doação de sangue. Andaleeb \& Basu 8 testaram variáveis atitudinais diretamente relacionadas com a doação de sangue. Essas variáveis atitudinais incorporam três aspectos: (a) a confiança individual na unidade hemoterápica; (b) medo pessoal de adquirir problemas de saúde pelo ato de doar; (c) medo geral que as pessoas associam à doação de sangue. Neste trabalho foi evidenciada a importância da confiança do doador no atendimento prestado. Segundo os autores, se as pessoas não confiam nos bancos de sangue, as im- plicações dessa atitude podem afetar o suprimento de sangue 8 .

O esforço de marketing da instituição, portanto, é transformar a primeira doação voluntária em doação contínua. Isto é, aumentar gradualmente as doações voluntárias e espontâneas, com conseqüente fidelização do doador 1 .

Nonis et al. 3 verificaram a possibilidade de existir uma diferença significativa entre doadores e não-doadores quanto ao risco percebido. Analisaram a influência dos fatores de risco físico (AIDS etc.), psicológico (medo), social (responsabilidade moral) e de tempo (falta de tempo) na decisão de doar sangue. Melnyk et al. 9 observaram a eficácia do layout no processo de doação, considerando a relevância dos fatores físicos da arquitetura interior de um banco de sangue na decisão dos doadores. Ludwig 6 pesquisou os tributos importantes da doação voluntária de sangue através da identificação das características pessoais dos doadores, dos aspectos ambientais e dos aspectos da interação doador-instituição que podem influenciar a doação. Gonçalez et al. 4 estudaram o perfil dos doadores de sangue após adoção de estratégias mercadológicas. Valdés et al. 10 investigaram os conhecimentos, atitudes e experiências sobre doação de sangue, para descobrir as barreiras que limitam a doação e os canais mais eficientes para desenvolver um programa de doação mais efetivo.

Nos estudos citados, é observada a importância da confiança do doador no atendimento prestado. Enfatiza-se neste ponto que a confiabilidade do serviço (o desempenho confiável e preciso do serviço) é o coração da excelência do marketing de serviços. Do ponto de vista do usuário, a prova de um serviço é sua realização impecável. Essa percepção é importante na medida em que se assenta sobre o que é percebido pelo doador, podendo ser descrita como o julgamento deste sobre a excelência do serviço 11 .

As pesquisas analisadas mostram a nova tendência das unidades hemoterápicas de utilizar instrumentos de marketing para que seja possível a expansão da base de doadores de sangue. As informações sobre os doadores são importantes para os colaboradores e administração, propiciando o conhecimento dos atributos considerados pelos doadores.

\section{Atributos importantes para a doação de sangue}

Leibrecht et al. 12 enfatizam que a necessidade cada vez maior de sangue tem despertado o interesse dos pesquisadores para descobrir as ra- 
zões que levam as pessoas a doar sangue. O desenvolvimento de estudos junto aos doadores de sangue vem evoluindo significativamente. Pesquisadores continuam buscando descobrir os motivos e atributos que levam pessoas a doar 13,14,15,16,17,18,19,20,21,22,23,24,25,26. Por outro lado, busca-se a construção de um relacionamento fiel com o doador, oferecendo um conjunto de benefícios que os doadores considerem valiosos, a ponto de retornar voluntariamente em outras ocasiões 1 .

Entre os aspectos examinados estão as variáveis demográficas, características de personalidade, incentivos e influências sócio-culturais 4,10,14,21,22,23,26. Segundo Andaleeb \& Basu 8, o desafio maior das instituições de saúde é manter e aumentar o conjunto dos doadores de sangue.

Segundo Oswalt 27 existem vários aspectos que levam o indivíduo a doar sangue: (a) altruísmo/humanitarismo; (b) programas de garantia ou pré-depósito; (c) reposição; (d) pressão social; (e) necessidades da comunidade; (f) recompensa; e (g) publicidade. Para Mauss $28 \mathrm{o}$ mais significativo desses aspectos é o altruísmo (amor ao próximo, filantropia) e a dádiva (oferta, donativo, presente), ato motivado por outros valores que não o lucro, a competitividade ou o sucesso. O ato de doar auxilia a humanidade e é uma ajuda para aqueles que estão necessitados. Mauss 28, nesse sentido, comenta a moral da dádiva-troca e enfatiza os fenômenos da vida econômica, citando que do mesmo modo que as dádivas não são livres, não são tão desinteressadas. Este comentário remete, à busca dos motivos, da causa da doação voluntária de sangue.

Breckler \& Wiggins 29 sugerem que a teoria de atitude tem muito a contribuir para a compreensão do comportamento e das razões do doador de sangue. Uma atitude é geralmente definida como uma predisposição para responder a um objeto dado de maneira favorável ou desfavorável. Uma suposição comumente aceita é que as atitudes variam ao longo de uma dimensão única de positiva para negativa. Entretanto, análises empíricas e teóricas da estrutura das atitudes identificaram três componentes distintos: sentimento, experiência e comportamento. Dessa maneira, uma atitude favorável à doação de sangue implica uma variedade de respostas positivas, incluindo as afetivas (emoções positivas associadas ao ato de doar), cognitivas (crenças, idéias e percepções favoráveis) e de procedimentos (recrutamento dos doadores e o ato de doar sangue). Aplicações prévias da teoria da atitude em estudos do comportamento do doador de sangue têm sido guiadas pela suposição de que a decisão para doar ou não doar sangue é cuidadosamente debatida 29 .

Ownby et al. 30 relatam que a idade e o nível de educação são os indicadores mais fortes da alta freqüência de doações e do retorno mais rápido. Citam, ainda, que a relação entre a causa do intervalo das doações e os futuros doadores pode comprovar a importância de compreender as razões das pessoas para retornar, possibilitando o desenvolvimento do recrutamento dos doadores e a estratégia de retenção.

Gonçalez et al. 4 descreveram os esforços realizados para encorajar a doação voluntária e os resultados obtidos demonstraram que a população foi sensível às campanhas efetuadas. Os autores sugerem esforços coordenados de marketing para aumentar a doação voluntária e contínua de sangue.

Ludwig ${ }^{6}$ verificou que as pessoas se sensibilizam com a necessidade de sangue, demonstrando disposição para doar quando efetivamente chamadas. Ainda, comenta que além da segurança quanto ao material descartável, os doadores enfatizam sobremaneira o atendimento por parte dos colaboradores. Dessa forma, destaca que a tranqüilidade que é passada, o tratamento recebido e o conhecimento e habilidades demonstradas pela equipe são aspectos altamente considerados pelos doadores.

Qualquer pessoa considera atributos quando faz uma escolha. A identificação e avaliação desses atributos orientarão, por conseqüência, a instituição a buscar esse doador. Porém, do mesmo modo que existem razões para doar, existem também as razões para não doar, o que, dentro deste contexto, destaca-se devido à influência que exercem junto aos possíveis doadores.

\section{Razões para não doar sangue}

Embora a questão principal seja definir por que as pessoas doam sangue, poucas pesquisas perguntam aos não-doadores quais as razões para não doar. Pesquisadores da saúde e das ciências do comportamento têm tentado, de várias maneiras, explicar o que diferencia o doador do não-doador. Oswalt 27 ao abordar essa questão enfatiza os aspectos negativos, isto é, as razões mais largamente utilizadas para não doar sangue: (a) medo; (b) desqualificação médica; (c) reações à doação; (d) apatia; e (e) conveniência.

Leibrecht et al. 12 sugerem que o ato de doar sangue representa uma aproximação e uma fuga, um conflito: para o doador ativo, para o doa- 
dor potencial e para o não-doador. Citam que existem aspectos negativos e aspectos positivos associados ao ato de doar sangue. Para o doador ativo, a importância dos aspectos positivos é maior do que os aspectos negativos, enquanto que para o não-doador é o contrário. Então, para ter sucesso no recrutamento dos doadores, a sugestão seria mudar a balança dos aspectos negativos para positivos, de modo que os positivos predominem.

Nesse sentido, os autores sustentam que as campanhas de informação deveriam enfatizar os aspectos positivos neutralizando os aspectos negativos. Um passo especialmente importante para transformar o não-doador em doador seria a concentração de esforços durante a primeira doação e o uso desta oportunidade para a conversão destes em doadores regulares, através da persuasão, do atendimento e das informações prestadas a respeito de todos os procedimentos 12 .

Porém, muitas perguntas permanecem sem respostas na área dos atributos motivacionais relacionados à doação de sangue. A meta final, certamente, é a melhoria das técnicas de recrutamento de doadores; o aumento do número de doadores e o conhecimento das razões dos não-doadores 12. Entretanto, busca-se ressaltar a influência da confiança do doador na instituição, enfatizando a interação de serviço como fator atenuante das dúvidas e/ou medos do doador.

\section{Confiança na unidade hemoterápica}

A confiança na unidade hemoterápica pode ser influenciada de diversas maneiras. A perícia percebida e a intenção de outro grupo, cujas ações influenciam o resultado do grupo focado podem influenciar fortemente a confiança 6 . Por exemplo, se doadores potenciais acreditam na falta de habilidade dos colaboradores, a confiança diminuirá. Os doadores também podem acreditar que os colaboradores não são responsáveis o bastante para protegê-los de possíveis danos, tais como: agulhas não-esterilizadas, erros ou enganos nos testes sorológicos e outros procedimentos. Essa possibilidade de negligência pode atenuar a confiança e, conseqüentemente, afetar a doação 8 .

Andaleeb \& Basu 8 constataram que a propensão para correr riscos diferencia o doador do não-doador. Uma variável encontrada na pesquisa, a força da explicação, foi gerada pela percepção dos riscos e pela confiança depositada nas unidades hemoterápicas, demonstrando que pode haver por meio desta o incre- mento em confiança e redução dos riscos percebidos com a saúde.

Ludwig 6 explicita que a confiança é um fator importante em decisões de doar sangue, contudo salienta que as pessoas têm idéias diferentes em relação ao que inspira confiança. Desta forma, a ênfase no atendimento pode fazer diferença no retorno do doador voluntário. Pois, quanto maior o nível de confiança que os indivíduos têm na instituição é mais provável que venham doar. Porém, mesmo que as pessoas confiem, pode ser que tenham interesse em saber os danos potenciais que podem ocorrer devido à incompetência ou má vontade dos colaboradores. Assim, o medo de adquirir problemas de saúde pode ser atribuído a aspectos além do controle da unidade hemoterápica e da confiança gerada por seus colaboradores.

Em seus esforços para aumentar a doação, as instituições têm tradicionalmente apelado aos instintos altruísticos dos doadores potenciais e o resultado dos estudos de Andaleeb \& Basu 8 indicam que os bancos de sangue deveriam aumentar os escopos de seu esforço. Os autores exemplificam que o relacionamento com confiança e risco de saúde percebido tem implicações administrativas significantes e sugerem um curso claro de ação: (a) passos estudados para reduzir o risco associado com a doação e criação de uma atmosfera de confiança; (b) levar informações sobre doação de sangue ao público. Os autores citam que a primeira tarefa representa uma filosofia continuada de operação em vez de uma meta de curto prazo. Comentam, ainda, que essa tarefa requer planejamento e o envolvimento ativo dos colaboradores de qualquer nível da organização. Enfatizam a necessidade de uma perspectiva planejada devido à importância da confiança nas decisões de doar.

As instituições também deveriam continuamente rever seus procedimentos, estabelecendo e mantendo padrões de qualidade, monitorando e guiando o comportamento dos colaboradores para acentuar o interesse pelo doador. Esse procedimento é importante, pois se reconhece que o doador forma um julgamento global da operação, baseado no que percebe e observa: limpeza, ambiente, profissionais, comportamento prestativo e cortês dos colaboradores para com os doadores, exatidão dos procedimentos e tempo do serviço. Esses aspectos desenvolvem a confiança do doador. Uma vez que a unidade hemoterápica confia nas suas práticas, deve partir para a segunda tarefa e empreender comunicações com o público de maneira a reduzir a percepção sobre o risco de saúde e alimentar a confiança 6 . 
Andaleeb \& Basu ${ }^{8}$ procuraram entender os atributos que influenciam a decisão de doar sangue e concluíram que pessoas mais velhas doam mais que as pessoas mais moças porque o senso de responsabilidade social aumenta com a idade, porém citam que para incrementar a doação há a necessidade de reduzir os riscos associados à doação e criar uma atmosfera de confiança. Melnyk et al. ${ }^{9}$ acompanharam a receptividade dos doadores para a arquitetura interior do Centro Lansing da Região dos Grandes Lagos da Cruz Vermelha Americana (Lansing Center of the Great Lakes Region of the American Red Cross - ACR) e observaram a satisfação dos doadores com os procedimentos do processo de coleta de sangue.

Ownby et al. 30 demonstraram que os doadores de sangue doam com um sentimento de dever, pressão e/ou benefícios pessoais e/ou familiares. Além disso, citaram que a falta de informações e o medo são os principais aspectos desencorajadores. Ludwig 6 verificou que a utilização de material descartável e os aspectos de segurança, confiança e atendimento destacaram-se como atributos importantes de uma unidade hemoterápica. Da mesma forma, identificou que a grande maioria dos doadores doa sangue para alguém das suas relações, sendo que menos da metade destes nunca doou sangue antes. Este aspecto demonstra que, apesar de haver essa necessidade por parte das instituições, não existe um fator motivacional mais concreto que a solicitação de amigos ou parentes. Dessa forma, constata-se que os resultados evidenciam, entre outros, a importância da qualidade do atendimento e da confiança na diminuição dos medos e da percepção do risco de doar.

\section{Considerações finais}

A preocupação com o relacionamento, com a fidelização dos doadores e, principalmente, com o estabelecimento de relações mais fortes deveria ser tarefa primordial das instituições. De acordo com Vavra 31, a oferta apropriada de serviços pode ser estabelecida à parte da rotina de interação e consiste de quatro componentes: espontaneidade, sinceridade, reconhecimento e consistência. No entanto, ainda que detalhadamente planejado, à medida que os usuários são identificados e diferenciados a interação torna-se importante para a correta adequação dos serviços às necessidades individuais dos doadores.

Como a confiança na unidade hemoterápica é influenciada de diversas maneiras, a per- cepção dos conhecimentos e perícia dos colaboradores influencia fortemente esse aspecto. Por isso, a ênfase no atendimento pode fazer diferença no retorno do doador voluntário de sangue. De acordo com Cobra 32 todo atendimento é composto de uma lista de atributos: rapidez, cordialidade, conhecimento, solução de problemas e assim por diante.

Dessa maneira, a ênfase nessa questão torna-se importante, pois configura a possibilidade de antecipar ações orientadas para os doadores. A comunicação é uma parte substancial da função de marketing. Existe uma conexão com a maneira como a qualidade do serviço é percebida, pois os efeitos da comunicação junto com outros fatores moldam a imagem da instituição na mente dos doadores. Esta acepção vai ao encontro do estudo realizado por Gonçalez et al. 4 .

Da mesma forma, esse aspecto alinha-se às conclusões de Parasumaran et al. 33 que destacam a complexidade do processo de percepção do usuário e enfatizam a qualidade funcional, levando em consideração as percepções do usuário em relação ao serviço prestado considerando, entre outros aspectos, a tangibilidade. Pois, o que conta de fato é a percepção que o doador tem da prestação do serviço ${ }^{11}$.

Como a exemplo de outras abordagens de marketing, uma campanha de doação de sangue deve ser planejada, testada e medida, sendo necessário, ainda, o acompanhamento por ações concretas de educação e assistência por algum tempo. Devido ao fato de que a doação de sangue não está inserida nos hábitos de grande parte da população brasileira, a inserção da doação nos hábitos e crenças sociais não será um processo rápido, pois o ser humano tende a ser resistente a mudanças.

$\mathrm{Na}$ verdade, talvez o maior imperativo que se coloca para as instituições seja a capacidade de divulgar para a população em geral a importância de aumentar a segurança de quem recebe o sangue doado. A doação de sangue salva vidas, porém, a doação contínua requer exames rotineiros, o que aumenta a margem de segurança quanto a doenças transmissíveis. Desta forma, observa-se que para o futuro deve-se pensar não mais em termos de reposição de sangue, mas sim de transfusões de sangue com maior margem de segurança. Isto pode ser obtido através de doadores voluntários e contínuos.

Entretanto, o processo de adoção de novos métodos e soluções é um desafio constante em marketing e temas mais complexos desafiam tabus sociais. A evolução da abordagem de marketing indiferenciada, na tentativa de atingir toda sociedade pode ser desenvolvida a partir 
da análise dos segmentos e grupos sociais e de suas características diferenciais, aumentando sua eficácia. De acordo com Nonis et al. 3 o segmento dos doadores potenciais e o segmento dos não-doadores mostram características e procedimentos diferentes. Mesmo assim, os autores enfatizam que é mais efetivo direcionar as estratégias para o conjunto da população.

O reconhecimento da importância das ações institucionais e da orientação para o usuário é fundamental quando se pensa em estratégia de marketing. Por meio dessa direção as instituições podem tentar identificar as reais necessidades e desejos da população.

Deve-se destacar, ainda, que um elemento comum às organizações é o aspecto de levarem em conta, de maneira primordial, o foco no usuário. Segundo Cobra 32 , descobrir em que a instituição pode beneficiar o usuário é o segredo que lhe permitirá dar um passo adiante na preferência deste. Cabe, entretanto, ter presente que o permanente monitoramento dos doadores deve ser enfatizado, pois se sabe que o ato de avaliar é inerente ao ser humano e que a necessidade de racionalizar condiciona as pessoas a uma avaliação objetiva. Pode-se, ainda, citar as limitações decorrentes da natureza do ser humano, o que dificulta sobremaneira a descoberta dos verdadeiros motivos, individuais, responsáveis pela tomada da decisão de doar sangue.

\section{Resumo}

Este trabalho enfatiza como o marketing pode fazer diferença no retorno do doador de sangue voluntário, pois o maior desafio enfrentado pelas instituições de saúde é manter e incrementar a doação de sangue. Nesse contexto, compreender a motivação do doador voluntário é de suma importância e os estudos apresentados demonstram que diversas variáveis são importantes na doação de sangue. O grande número de pacientes necessitados de transfusão de sangue e a carência de sangue no setor justificam o interesse por este estudo, não só do ponto de vista do doador, mas, sobretudo, das instituições. Além disso, o reconhecimento da importância das ações e da orientação para o doador é fundamental quando se pensa em estratégia de marketing. Dessa maneira, torna-se relevante para as instituições de saúde a identificação das reais necessidades e desejos do doador.

Doadores de Sangue; Marketing Social; Estratégias

\section{Colaboradores}

S. T. Ludwig coletou e revisou os dados da pesquisa, realizou as tabulações e análises, redigiu a versão preliminar do trabalho e realizou a edição e revisão da versão final do artigo. A. C. M. Rodrigues participou nas análises e revisão do artigo.

\section{Referências}

1. Agência Nacional de Vigilância Sanitária. Política Nacional de Sangue e Hemoderivados do Ministério da Saúde. http://www.anvisa.gov.br/divulga/noticias.htm (acessado em 10/Jul/2004).

2. Gianesi IGN, Correa HL. Administração estratégica de serviços: operações para satisfação do cliente. São Paulo: Atlas; 1994.

3. Nonis SA, Ford CW, Logan L, Hudson G. College student's blood donation behavior: relationship to demographics, perceived risk, and incentives. Health Mark Q 1996; 13:33-46.

4. Gonçalez T, Sabino EC, Chamone DF. Trends in the profile of blood donors at a large blood center in the city of São Paulo, Brazil. Rev Panam Salud Publica 2003; 13:144-8.

5. Ministério da Saúde. Formulação da Política Nacional de Sangue e Hemoderivados. http:/ / www. saude.gov.br/sps/daps/sangue/projetos/ (acessado em 10/Abr/2000).

6. Ludwig ST. Um estudo da doação voluntária de sangue em hospitais de Porto Alegre, RS [Dissertação de Mestrado]. Porto Alegre: Faculdade de Administração, Contabilidade e Economia, Pontifícia Universidade Católica do Rio Grande do Sul; 2001.

7. Solomon MR, Surprenant C, Czepiel JA, Gutman EG. A role theory perspective on dyadic interactions: the service encounter. J Mark 1985; 49:99111. 
8. Andaleeb SS, Basu AK. Explaining blood donation: the trust factor. J Health Care Mark 1995; 15:42-8.

9. Melnyk SA, Pagell M, Jorac G, Sharpe AS. Applying survival analysis to operations management: analyzing the differences in donor classes in the blood donation process. Journal of Operations Management 1995; 13:339-56.

10. Valdés MEA, Díaz RML, Santovenia JMB, Fonticoba WC, Planes LB, Breijo CJM, et al. Aspectos socioculturales relacionados con la donación de sangre en Cuba. Rev Cubana Hematol Inmunol Hemoter 2002; 18. http://bvs.sld.cu/revistas/hih/ vol18_3_02/hih08302.htm (acessado em 02/Dez/ 2004).

11. Rodrigues ACM. Qualidade de serviços e satisfação do consumidor: aspectos conceituais distintivos. Análise 2001; 12:175-88.

12. Leibrecht BC, Hogan JM, Luz GA, Tobias KI. Donor and nondonor motivations. Transfusion 1976; 16:182-9.

13. Belda-Suárez IM, Fernández-Montoya A, Rodriguez-Fernández A, López-Berrio A, Cillero-Peñuela M. How regular blood donors explain their behavior. Transfusion 2004; 44:1441-6.

14. Carrazzone CFV. Avaliação dos perfis sorológico e sócio-demográfico dos receptores de sangue do Hospital Universitário Oswaldo Cruz da Universidade de Pernambuco-UPE. Recife: Centro de Pesquisas Aggeu Magalhães, Fundação Oswaldo Cruz; 2004.

15. Ferguson E. Conscientiousness, emotional stability, perceived control and the frequency, recency, rate and years of blood donor behaviour. $\mathrm{Br} \mathrm{J}$ Health Psychol 2004; 9:293-314.

16. Carden R, DelliFraine JL. An examination of hospital satisfaction with blood suppliers. Transfusion 2004: 44:1648-55.

17. Cruz JR, Pérez-Rosales MD. Availability, safety, and quality of blood for transfusion in the Americas. Rev Panam Salud Publica 2003; 13:103-10.

18. Schreiber GB, Sanchez AM, Glynn SA, Wright DJ, Retrovirus Epidemiology Donor Study. Increasing blood availability by changing donation patterns. Transfusion 2003; 43:591-7.

19. Glynn SA, Williams AE, Nass CC, Bethel J, Kessler D, Scott EP, et al. Attitudes toward blood donation incentives in the United States: implications for donor recruitment. Transfusion 2003; 43:7-16.
20. Garay-Uriarte A, Íniguez-Rueda L, Martínez-González M, Muñoz-Justicia J, Pallarés-Parejo S, Vázquez-Sixto F. Evaluación cualitativa del sistema de recogida de sangre en Cataluña. Rev Esp Salud Publica 2002; 76:437-50.

21. Boulware LE, Ratner LE, Ness PM, Cooper LA, Campbell-Lee S, LaVeist TA, et al. The contribution of sociodemographic, medical, and attitudinal factors to blood donation among the general public. Transfusion 2002; 42:669-78.

22. Glynn SA, Kleinman SH, Schreiber GB, Zuck T, McCombs S, Bethel J, et al. Motivations to donate blood: demographic comparisons. Transfusion 2002; 42:216-25.

23. Wu Y, Glynn SA, Schreiber GB, Wright DJ, Lo A, Murphy EL, et al. First-time blood donors: demographic trends. Transfusion 2001; 41:360-4.

24. Sanchez AM, Ameti DI, Schreiber GB, Thomson RA, Lo A, Bethel J, et al. The potential impact of incentives on future blood donation behavior. Transfusion 2001; 41:172-8.

25. Kloetzel K, Bertoni AM, Irazoqui MC, Campos VPG, Santos RN. Controle de qualidade em atenção primária à saúde. I A satisfação do usuário. Cad Saúde Pública 1998; 14:263-8.

26. Castro-Santos LA. Doação, transfusão e laços de sangue: cultura e sociedade no Brasil contemporâneo. Hist Cien Saude-Manguinhos 1995; 2:16770.

27. Oswalt RM. A review of blood donor motivation and recruitment. Transfusion 1977; 17:123-35.

28. Mauss M. Ensaio sobre a dádiva. Lisboa: Edições 70; 2001.

29. Breckler SJ, Wiggins EC. Scales for the measurement of attitudes toward blood donation. Transfusion 1989; 29:401-4.

30. Ownby HE, Kong F, Watanabe K, Tu Y, Nass CC. Analysis of donor return behavior. Retrovirus Epidemiology Donor Study. Transfusion 1999; 39: 1128-35.

31. Vavra TG. Marketing de relacionamento: after marketing, como manter a fidelidade dos clientes através do marketing de relacionamento. São Paulo: Atlas; 1993.

32. Cobra M. Estratégias de marketing de serviços. São Paulo: Cobra; 2001.

33. Parasumaran A, Zeithaml V, Berry L. Communication and control process in the delivery of service quality. J Mark 1988; 52:35-48.

Recebido em 21/Jan/2004

Versão final reapresentada em 17/Dez/2004

Aprovado em 04/Jan/2005 